\title{
Incidence of inadvertent intercostal or epidural spread during thoracic sympathetic ganglion block
}

Received June 19, 2020

Revised July 16, 2020

Accepted July 17, 2020

\section{Corresponding author}

Ji Hee Hong, M.D., Ph.D.

Department of Anesthesiology and

Pain Medicine, Keimyung University

Dongsan Hospital, 1095 Dalgubeol-

daero, Dalseo-gu, Daegu 42601,

Korea

Tel: 82-53-258-7767

Fax: 82-53-258-6288

E-mail: swon13@daum.net

\section{Ji Hee Hong, Seung Won Yi, and Ji Seob Kim}

Department of Anesthesiology and Pain Medicine, Keimyung University Dongsan Hospital, Daegu, Korea

Background: Sympathetic blocks (SBs) have been used widely to relieve the symptoms of sympathetically maintained pain (SMP). The thoracic sympathetic ganglion is not separated from somatic nerves by muscles and connective tissue. The upper thoracic ganglion runs along the posterior surface of the vertebral column in close proximity to the adjacent epidural region. This anatomical difference leads to frequent epidural and intercostal spread in cases of thoracic SBs. The purpose of this study was to investigate the incidence of inadvertent intercostal and epidural injections during thoracic SBs.

Methods: Twenty-two patients who were suffering from complex regional pain syndrome or lymphedema after breast cancer surgery were managed with two or three times of thoracic SBs. Therefore, injections of 63 thoracic SBs from 22 patients were enrolled in this study. An investigator who did not attend the procedure evaluated the occurrence of intercostal or epidural spread using anteroposterior fluoroscopic images.

Results: The overall incidence of inadvertent intercostal or epidural spread of contrast was 47.5\%. Among the inadvertent injections, intercostal spread (34.9\%) was more frequent than epidural spread (12.6\%). Only $52.5 \%$ of the thoracic SBs demonstrated successful contrast spread without any inadvertent spread. The mean difference in skin temperature between the blocked and unblocked sides was $2.5 \pm 1.8^{\circ} \mathrm{C}$. Fifty-nine (93.6\%) injections demonstrated more than $1.5^{\circ} \mathrm{C}$ difference.

Conclusions: Thoracic SBs showed a high incidence (47.5\%) of inadvertent epidural or intercostal injection. Thus, special attention is required for the diagnosis of SMP or the injection of any neurolytic agent around sympathetic ganglion.

Keywords: Anatomical difference; Inadvertent injection; Injection, epidural, intercostal; Skin temperature; Sympathetic blocks; Sympathetically maintained pain; Thoracic sympathetic ganglion.

\section{INTRODUCTION}

The autonomic nervous system includes a sympathetic, a parasympathetic, and an enteric nervous system which provide the neural control of all parts except for skeletal muscles. The sympathetic and parasympathetic nervous system are differentiated in structure and function from the remainder of the peripheral components. The sympathetic

This is an Open Access article distributed under the terms of the Creative Commons Attribution Non-Commercial License (http://creativecommons.org/licenses/by-nc/4.0) which permits unrestricted non-commercial use, distribution, and reproduction in any medium, provided the original work is properly cited.

Copyright (C) the Korean Society of Anesthesiologists, 2020 
trunk, extending from the base of the skull to the coccyx, presents bilaterally. It divides into the cervical, thoracic, lumbar and sacral segments [1-3].

Certain neuropathic pain conditions are referred to as sympathetically maintained pain (SMP). Although the mechanism of SMP is still unclear, abnormal coupling between the sympathetic and the somatosensory nervous system has been suggested. This coupling occurs as a result of neurogenic inflammation following a lesion in the peripheral nerve or the dorsal root ganglion [4-6].

Clinically, sympathetic blocks (SB) have been widely used to relieve the symptoms of SMP or to differentiate between SMP and sympathetically-independent pain. For an SB to provide a diagnostic value, the sympathetic activity should be successfully disturbed for a proper duration of time [7-9]. For the diagnosis of SMP using an SB, complete interruption of sympathetic activity must be achieved, while preserving sensory and motor function. The sympathetic trunk at the lumbar region runs on the anterolateral surface of the vertebral column from the L1 to L4 levels and deep to the medial aspect of the psoas major muscle [10]. Therefore, it is hard to find epidural contrast spread during lumbar SB due to the anterior location of lumbar sympathetic ganglion to the vertebral body. Our previous study demonstrated frequent psoas muscle injection due to the close proximity of lumbar sympathetic ganglion [11].

In contrast to the lumbar sympathetic ganglion, the thoracic sympathetic ganglion is not separated from somatic nerves by muscles and connective tissue. Moreover, the upper thoracic ganglion runs along the posterior surface of the vertebral column in close proximity to adjacent epidural region [12]. These anatomical differences in the thoracic sympathetic ganglion lead to frequent epidural and intercostal spread when performing thoracic SBs. Spread to the epidural and intercostal space lowers the diagnostic and therapeutic value of thoracic SBs. In addition, serious adverse outcomes can be encountered if a neurolytic agent such as alcohol is injected into the epidural or intercostal space inadvertently during the procedure of thoracic SB. Considering the diagnostic and therapeutic value and safety of thoracic SBs, evaluation of the actual incidence of intercostal and epidural spread is important.

The conventional target point of a thoracic SB in lateral fluoroscopic images is the anterior edge of the costovertebral articulation, where the needle tip contacts the posterior one-third of the lateral vertebral body $[9,13]$.

The purpose of this study was to evaluate the incidence of intercostal and epidural spread when the needle tip was located at the conventional target point.

\section{MATERIALS AND METHODS}

\section{Patients}

This prospective randomized study was approved by the Institutional Review Board (no. 05-028-004) of our institution. All participants were explained about potential benefits and risks of the trial and they were provided with written informed consent. This trial was registered prior to patient enrollment at ClinicalTrials.gov (no. NCT03995576, Date of registration: 06/20/2019).

From June to August 2019, 22 patients who were managed with two or three times of thoracic SBs were enrolled. The inclusion criteria in this study were patients who demonstrated severe unilateral arm pain or edema due to complex regional pain syndrome (CRPS) or lymphedema after breast cancer surgery. The enrolled patients were intractable to conservative therapy, including pain medication and physical therapy. Patients who had known allergies to contrast or local anesthetics, or who had coagulopathies or spine infections, were excluded. Therefore, the ultimate enrolled number of the thoracic SBs was 63 injections. All procedures were performed by one pain physician who had more than 15-years of experience with pain intervention using fluoroscopy.

\section{Procedure}

In the thoracic SB procedure, the patient was laid on the fluoroscopic bed in a prone position and the upper back of the target vertebra was sterilized. To facilitate needle insertion, fluoroscopy was rotated ipsilaterally by $15-20^{\circ}$. After local infiltration with $1 \%$ lidocaine, a 10 -cm long, 23-gauge spinal needle was inserted medially toward the lateral margin of the second or third thoracic vertebra. Strict attention was paid to insert the needle within a $3-\mathrm{cm}$ distance from the spinous process of the targeted vertebra to minimize the risk of pneumothorax. A right or left side injection was determined according to the symptomatic side of pain or edema.

A spinal needle was inserted using a tunnel view technique under oblique view. After the needle touched the lateral vertebral body, it was slightly advanced to the posterior one-third of the vertebral body. The final location of 
the needle tip was confirmed using anteroposterior (AP) and lateral fluoroscopic views.

After the needle tip was located successfully, $3 \mathrm{ml}$ of contrast medium (omnipaque 300, GE Healthcare, UK) was injected slowly. All AP and lateral images were saved to the hard disc of the fluoroscopic machine and were transmitted to a picture archiving and communication system (INFINITT Healthcare, Korea).

\section{Outcome measurements}

Age, sex, diagnosis of patient, and skin temperature of both hands before and after thoracic SB were collected from the medical record.

Temperature was measured for $20 \mathrm{~min}$ at 5-min intervals using a round-shaped skin temperature sensor attached to the volar side of both thumbs (carescape monitor B650, GE Healthcare). If the temperature difference between the two fingertips was more than $1.5^{\circ} \mathrm{C}$, the sympathetic block was considered successful [14].

An investigator who did not attend the procedure evaluated the occurrence of intercostal or epidural spread using the AP images which were saved in a picture archiving and communication system. This investigator had more than 10-years of experience with pain intervention using fluoroscopy. Patterns of spread were divided into successful thoracic SBs without any inadvertent spread (Fig. 1A), thoracic SBs with intercostal spread (Fig. 1B), thoracic SBs with epidural spread (Fig. 1C) and failed SBs.

\section{Statistical analysis}

Our study is a simple observational study to report the incidence of epidural or intercostal spread. Therefore, we did not perform any power analysis to calculate the sample size. We obtained the incidence of inadvertent injections from 63 injections of thoracic SBs.

Continuous numerical data were expressed as mean and standard deviation (for normally distributed data). Categorical data were expressed as frequencies and percentages. Chi-square or Fisher's exact test was used for the categorical variables. All data were analyzed using SPSS version 18.0 (IBM Co., USA) and a P value < 0.05 was considered statistically significant.

\section{RESULTS}

This study included 63 thoracic SBs performed on 22 patients with a mean age of $42.5 \pm 6.5$ years. Among 22 patients enrolled, 18 patients had CRPS and 4 patients had lymphedema (Table 1).

Thoracic SBs were performed at T2 or T3 levels. Thir-

Table 1. Demographic Data

\begin{tabular}{lc}
\hline \multicolumn{1}{c}{ Variable } & Value \\
\hline Age $(\mathrm{yr})$ & $42.5 \pm 6.5$ \\
Sex $(\mathrm{M} / \mathrm{F})$ & $10 / 12$ \\
Body mass index $\left(\mathrm{kg} / \mathrm{m}^{2}\right)$ & $22.5 \pm 5.2$ \\
Type of disease & \\
Complex regional pain syndrome & 18 \\
$\quad$ Lymphedema & 4 \\
\hline
\end{tabular}

Values are presented as mean \pm SD or number of patients.
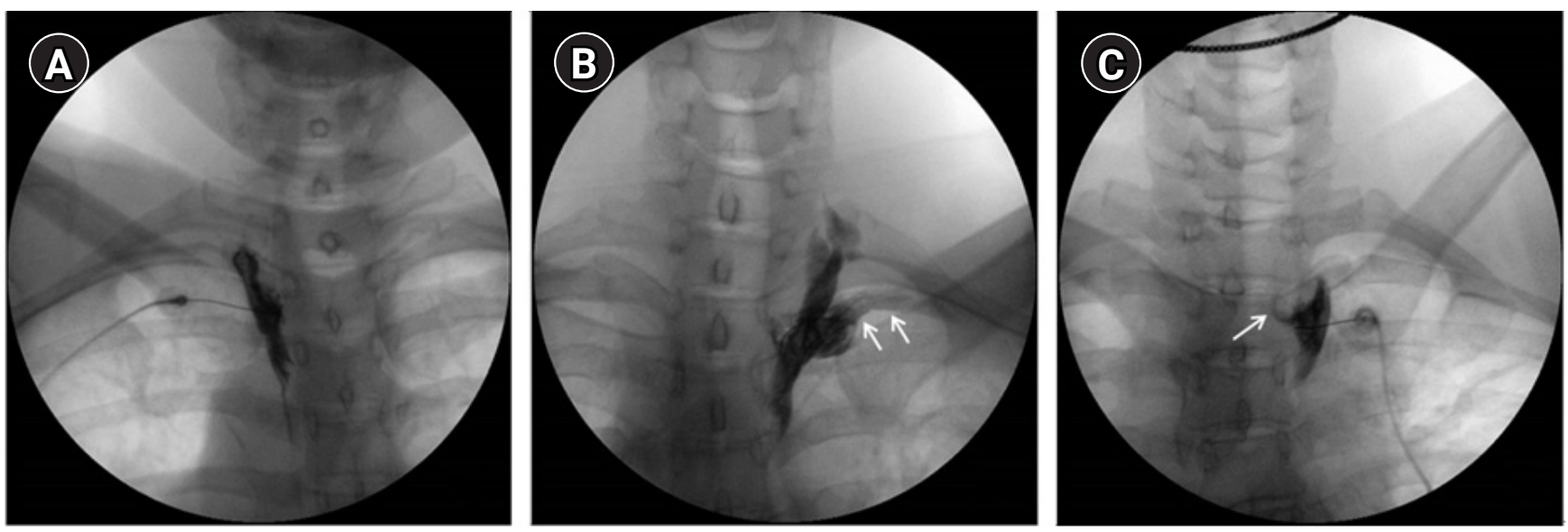

Fig. 1. Patterns of thoracic sympathetic block (SB) showing successful SB without any inadvertent spread (A), thoracic SB with intercostal spread (B), and thoracic SB with epidural spread (C). White arrows in (B) and (C) indicates intercostal and epidural spreads, respectively. 
Table 2. Incidence of Intercostal or Epidural Spread of Contrast Medium during Thoracic Sympathetic Ganglion Block

\begin{tabular}{|c|c|c|c|c|}
\hline \multirow{2}{*}{ Incidence } & \multicolumn{2}{|c|}{ Vertebral levels } & \multirow{2}{*}{ Total } & \multirow{2}{*}{$P$ value } \\
\hline & $\mathrm{T} 2$ & T3 & & \\
\hline Number of blocks & 34 & 29 & $63(100)$ & \\
\hline Intercostal spread of contrast & $14(22.2)$ & $8(12.6)$ & $22(34.9)$ & 0.682 \\
\hline Epidural spread of contrast & $6(9.5)$ & $2(3.1)$ & $8(12.6)$ & \\
\hline
\end{tabular}

Values are presented as number or mean \pm SD.

ty-one injections were made on the right side and 32 injections were made on the left side.

The overall incidence of inadvertent intercostal or epidural spread of contrast was $47.5 \%$. Among the inadvertent injections, intercostal spread (34.9\%) was more frequent than epidural spread (12.6\%). Only $52.5 \%$ of the thoracic SBs demonstrated successful contrast spread without any inadvertent spread (Table 2). There was no failed SB.

The right and left side thoracic SBs showed 15/31 (48.3\%) and 7/32 (21.8\%) inadvertent intercostal injections, respectively. Skin temperature was measured at both thumbs to assess the block efficacy (Fig. 2). The mean difference in skin temperature between the blocked and unblocked sides was $2.5 \pm 1.8^{\circ} \mathrm{C}$. Fifty-nine (93.6\%) injections demonstrated more than $1.5^{\circ} \mathrm{C}$ difference.

\section{DISCUSSION}

In this study, about half (47.5\%) of thoracic SBs showed inadvertent intercostal or epidural spread. Among the inadvertent injections, the intercostal spread of contrast was more frequent than epidural spread. When sympathetic blocks were performed at the lumbar area, the incidence of psoas muscle spread was $21.3 \%$ [11]. It is thought that the reason of more frequent inadvertent injection of thoracic SB than lumbar SB comes from the anatomical difference of the thoracic sympathetic ganglion. The upper thoracic ganglion runs along the posterior surface of the vertebral column in close proximity to the adjacent epidural region [12]. Due to this posterior location, thoracic SBs performed in this study targeted a final needle location at the posterior one-third of vertebral body.

During thoracic SB, the communicating ramus is the key structure by which injected medicine is delivered to the intercostal nerve. White and gray communicating rami are located between intercostal nerve and thoracic sympathetic ganglion. The types of communicating rami of the thoracic sympathetic ganglion include transverse or oblique rami connecting to the intercostal nerve of the same level,

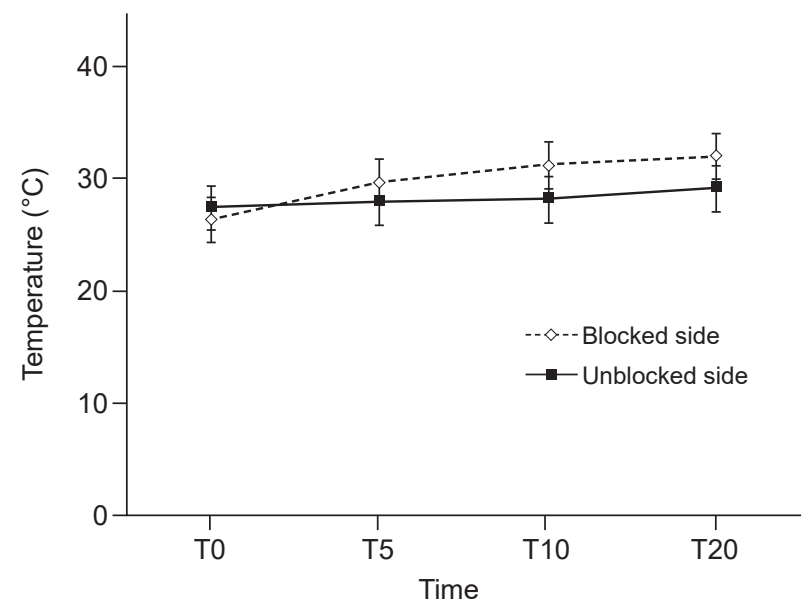

Fig. 2. Degree of skin temperature increase between blocked and unblocked side measured at volar side of thumb. Values are presented as mean \pm SD. TO: before thoracic sympathetic block (SB), T5: 5 min after thoracic SB, T10: 10 min after thoracic SB, T20: 20 min after thoracic SB.

ascending rami to the intercostal nerve of the higher level and descending rami to the intercostal nerve of the lower level $[12,15]$. The second thoracic sympathetic ganglion presents a diverse way of giving off its communicating rami. The incidence of ascending or descending rami arising from the second thoracic sympathetic ganglion was $66.7 \%$ and this incidence decreased below T3 level. In addition, T2 showed much stronger anatomical variation compared to T3 or T4 [15]. Considering this anatomical variation and the diverse way of giving off ascending or descending rami at T2 sympathetic level, a significant inadvertent incidence of T2 would be expected. However, this study did not show any significant differences between T2 and $\mathrm{T} 3$ levels.

Right-side thoracic SBs showed an increased incidence of inadvertent injections compared to the left side in this study. According to a recent cadaver study, the number of rami of the upper thoracic sympathetic chain was significantly greater on the right side. Also, the horizontal distance between the sympathetic chain and union of the rami on the intercostal nerves was significantly greater on the right side [15]. This anatomical difference of the upper 
thoracic sympathetic chain might contribute to the slightly increased inadvertent injections on right-side thoracic SBs.

Primary palmar hyperhidrosis is a debilitating disorder characterized by excessive sweating. The communicating rami of the upper thoracic sympathetic ganglion are frequently involved in essential palmar hyperhidrosis. A surgical method of dividing the sympathetic communicating rami, while preserving the thoracic sympathetic ganglia and nerve chain, has been introduced to address this disorder [16]. Percutaneous ethanol or alcohol sympatholysis is another treatment modality for primary hyperhidrosis. According to a recent study, 39 consecutive patients with primary hyperhidrosis received percutaneous sympatholysis with a technical success rate of $100 \%$. However, major complications were encountered, including severe intercostal neuralgia and pneumothorax [17]. Therefore, if chemical neurolysis will be performed using alcohol or ethanol, instead of a surgical approach, special attention should be paid to minimize inadvertent spread.

A stellate ganglion block is the most commonly used simple technique to interrupt the sympathetic innervation of the upper extremity [8]. However, clinical and anatomical studies have suggested that this may not be the best method for upper extremity sympathetic block $[7,9,18]$. Complex regional pain syndrome treated by thoracic SB showed reduced pain intensity with improved depression and quality of life [7,9]. Frequent occurrences of epidural and intercostal injections during thoracic SBs, leading to an unwanted somatic blockade, might affect the therapeutic outcome of complex regional pain syndrome.

This study included patients with lymphedema after breast cancer surgery. Our pain clinic performs thoracic SBs to manage an intractable upper limb edema occurring after breast surgery. A previous study showed reductions in arm circumferences with improved lymphedema and breast cancer questionnaire scores after thoracic SBs [19].

Our study had several limitations. In this study, skin temperature difference was measured to determine the property of thoracic SBs. However, the association between thoracic SBs, with or without inadvertent spread and skin temperature differences, was not evaluated. Further study is needed to determine whether the appearance of inadvertent spread during thoracic SB affects skin temperature or therapeutic outcome.

In addition, we did not present any technical method to reduce high incidence of inadvertent injection of thoracic SBs. Future studies on thoracic SBs, comparing new and conventional approaches, are required. Lastly, our study has some limitations due to small sample size. Further multicenter study with enough cases of thoracic SBs might present new method and has a higher impact.

In conclusion, inadvertent intercostal and epidural spreads, which lead to somatic blockade, were observed in half of the thoracic SB injections. Considering such a high incidence of inadvertent injections, injecting any neurolytic agent or diagnosing SMP should be performed cautiously.

\section{CONFLICTS OF INTEREST}

No potential conflict of interest relevant to this article was reported.

\section{AUTHOR CONTRIBUTIONS}

Conceptualization: Ji Hee Hong. Data curation: Ji Hee Hong. Methodology: Ji Hee Hong, Seung Won Yi. Writing-original draft: Ji Hee Hong, Seung Won Yi. Writing-review \& editing: Ji Hee Hong. Investigation: Ji Hee Hong. Supervision: Ji Hee Hong, Ji Seob Kim.

\section{ORCID}

Ji Hee Hong, https://orcid.org/0000-0002-6679-3088

Seung Won Yi, https://orcid.org/0000-0003-3079-4385

Ji Seob Kim, https://orcid.org/0000-0002-8273-8644

\section{REFERENCES}

1. Chaudhry A, Kamali A, Herzka DA, Wang KC, Carrino JA, Blitz AM. Detection of the stellate and thoracic sympathetic chain ganglia with high-resolution 3D-CISS MR imaging. AJNR Am J Neuroradiol 2018; 39: 1550-4.

2. Elias M. Cervical sympathetic and stellate ganglion blocks. Pain Physician 2000; 3: 294-304.

3. Wehrwein EA, Orer HS, Barman SM. Overview of the anatomy, physiology, and pharmacology of the autonomic nervous system. Compr Physiol 2016; 6: 1239-78.

4. Krumova EK, Gussone C, Regeniter S, Westermann A, Zenz M, Maier C. Are sympathetic blocks useful for diagnostic purposes? Reg Anesth Pain Med 2011; 36: 560-7.

5. Gibbs GF, Drummond PD, Finch PM, Phillips JK. Unravelling the pathophysiology of complex regional pain syndrome: focus on sympathetically maintained pain. Clin Exp Pharmacol Physiol 2008; 35: 717-24. 
6. Sommer C, Leinders M, Üçeyler N. Inflammation in the pathophysiology of neuropathic pain. Pain 2018; 159: 595-602.

7. Park J, Lee YJ, Kim ED. Clinical effects of pulsed radiofrequency to the thoracic sympathetic ganglion versus the cervical sympathetic chain in patients with upper-extremity complex regional pain syndrome: a retrospective analysis. Medicine (Baltimore) 2019; 98: e14282.

8. Zhu X, Kohan LR, Morris JD, Hamill-Ruth RJ. Sympathetic blocks for complex regional pain syndrome: a survey of pain physicians Reg Anesth Pain Med; 2019 doi: 10.1136/rapm2019-100418 [Epub ahead of print].

9. Rocha Rde O, Teixeira MJ, Yeng LT, Cantara MG, Faria VG, Liggieri $\mathrm{V}$, et al. Thoracic sympathetic block for the treatment of complex regional pain syndrome type I: a double-blind randomized controlled study. Pain 2014; 155: 2274-81.

10. Murata Y, Takahashi K, Yamagata M, Takahashi Y, Shimada Y, Moriya H. Variations in the number and position of human lumbar sympathetic ganglia and rami communicantes. Clin Anat 2003; 16: 108-13.

11. Hong JH, Kim AR, Lee MY, Kim YC, Oh MJ. A prospective evaluation of psoas muscle and intravascular injection in lumbar sympathetic ganglion block. Anesth Analg 2010; 111: 802-7.

12. Schiller Y. The anatomy and physiology of the sympathetic innervation to the upper limbs. Clin Auton Res 2003; 13 Suppl 1:
I2-5.

13. Kim WH, Lee CJ, Kim TH, Shin BS, Sim WS. The optimal oblique angle of fluoroscope for thoracic sympathetic ganglion block. Clin Auton Res 2011; 21: 89-96.

14. Schürmann M, Gradl G, Wizgal I, Tutic M, Moser C, Azad S, et al. Clinical and physiologic evaluation of stellate ganglion blockade for complex regional pain syndrome type I. Clin J Pain 2001; 17: 94-100.

15. Cho HM, Lee DY, Sung SW. Anatomical variations of rami communicantes in the upper thoracic sympathetic trunk. Eur J Cardiothorac Surg 2005; 27: 320-4.

16. Akil A, Semik M, Fischer S. Efficacy of miniuniportal video-assisted thoracoscopic selective sympathectomy (ramicotomy) for the treatment of severe palmar and axillar hyperhidrosis. Thorac Cardiovasc Surg 2019; 67: 415-9.

17. Brock M, Frangakis C, Georgiades CS. CT-guided, percutaneous ethanol sympatholysis for primary hyperhidrosis. Cardiovasc Intervent Radiol 2018; 41: 477-82.

18. Ramsaroop L, Partab P, Singh B, Satyapal KS. Thoracic origin of a sympathetic supply to the upper limb: the 'nerve of Kuntz' revisited. J Anat 2001; 199(Pt 6): 675-82.

19. Choi E, Nahm FS, Lee PB. Sympathetic block as a new treatment for lymphedema. Pain Physician 2015; 18: 365-72. 